Monatsschr Kinderheilkd 2019 · 167:46-50 https://doi.org/10.1007/s00112-017-0340-y Online publiziert: 11. Juli 2017 (c) Der/die Autor(en) 2017. Dieser Artikel ist eine Open-Access-Publikation., Dieser Artikel ist eine Open-Access-Publikation.

\section{Redaktion}

B. Koletzko, München

T. Lücke, Bochum

E. Mayatepek,Düsseldorf

N. Wagner, Aachen

S. Wirth, Wuppertal

F. Zepp, Mainz

CrossMark

\author{
A. Trobisch ${ }^{1}$ D. S. Klobassa $\cdot$ H. Gschiel $l^{2}$ M. Wassermann-Neuhold ${ }^{3} \cdot$ W. Zenz $^{1}$ \\ ${ }^{1}$ Ambulanz für Vakzinologie und Infektionskrankheiten, Klinische Abteilung für Allgemeine Pädiatrie, \\ Medizinische Universität, Graz, Österreich \\ ${ }^{2}$ Akademie für Vorsorgemedizin Graz, Graz, Österreich \\ ${ }^{3}$ Sanitätsdirektion, Amt der steiermärkischen Landesregierung, Graz, Österreich
}

\title{
Analyse des Masernausbruches 2015 in der Steiermark
}

\section{Masernverbreitung erfolgte durch Impfgegner}

\section{Einleitung}

Im Jahre 1998 hat die WHO die Ausrottung der Masern in Europa bis 2007 zum Ziel erklärt. 2014 gab die WHO Bescheid, dass die Frist zur Masern-Eradikation zum bislang dritten Mal auf 2015 verlängert werden musste [3]. Auch dieses Ziel wurde nicht eingehalten und so werden derzeit die Informationen der vergangenen Jahre analysiert, um weitere Empfehlungen herauszugeben [4].

Schmid et al. berichteten 2008 über einen Masernausbruch in Österreich, der insgesamt 259 Masernfälle beschrieb, wo bei 79 \% der Betroffenen eine Verbindung $\mathrm{zu}$ einer einzelnen anthroposophischen Schule in Salzburg bestand $[9,10]$.

2009 wurde ein Masernausbruch in der Steiermark mit 37 Erkrankten beschrieben, von denen 25 einer anthroposophischen Gemeinschaft angehörten und der ebenfalls seinen Ausgang an einer anthroposophischen Schule genommen hatte [6].

2014 war Österreich mit 13 Masernerkrankten pro 1.000.000 Einwohner an die 12. Stelle aller WHO-Länder gerückt [8].

2015 wurden in Österreich allein zwischen Januar und März 115 Masernfälle gemeldet. Die Anzahl der Masernfälle in Europa belief sich für diesen Zeitraum auf 1774 [2].

Diese Arbeit beschreibt einen Masernausbruch in der Steiermark mit 19 betroffenen Kindern oder Jugendlichen und über 200 exponierten Personen (davon waren 40 Säuglinge) und untersucht die
Rolle von Impfgegnern in der Verbreitung der Erkrankung.

\section{Methode}

Nach Auftreten der ersten Masernfälle erfolgte die prospektive und retrospektive Dokumentation aller Fälle im Zeitraum von Februar 2015 bis Mai 2015 an der Univ.-Klinikf. Kinder- u. Jugendheilkunde Graz. Die erhobenen Daten wurden mit den Meldedaten der steirischen Landessanitätsdirektion abgeglichen.

In weiterer Folge erfolgte der persönliche Kontakt der Erziehungsberechtigten aller Masernpatienten im Kindes- und Jugendalter, welche 2015 im Einzugsgebiet der Universitätskinderklinik Graz gemeldet wurden. Das Einzugsgebiet der Univ.Klinik für Kinder- u. Jugendheilkunde Graz umfasst hierbei 800.000 Personen, wovon in etwa 180.000 Kinder und Jugendliche sind [13].

Die Masernfälle galten als gesichert bei virologischem Nachweis (positive Polymerase chain reaction oder positive Masern-Immunglobulin M-Antikörper [Masern-IgM-AK]) oder als wahrscheinlich bei typischem klinischen Bild und Kontakt zu einem anderen an gesicherten Masern erkrankten Patienten im zur Inkubationszeit passenden Zeitraum.

Es wurde ein Vergleich mit der steirischen Impfdatenbank durchgeführt. In der steirischen Impfdatenbank der wissenschaftlichen Akademie für Vorsorgemedizin werden seit 1999 alle in der Steiermark durchgeführten Impfungen, 


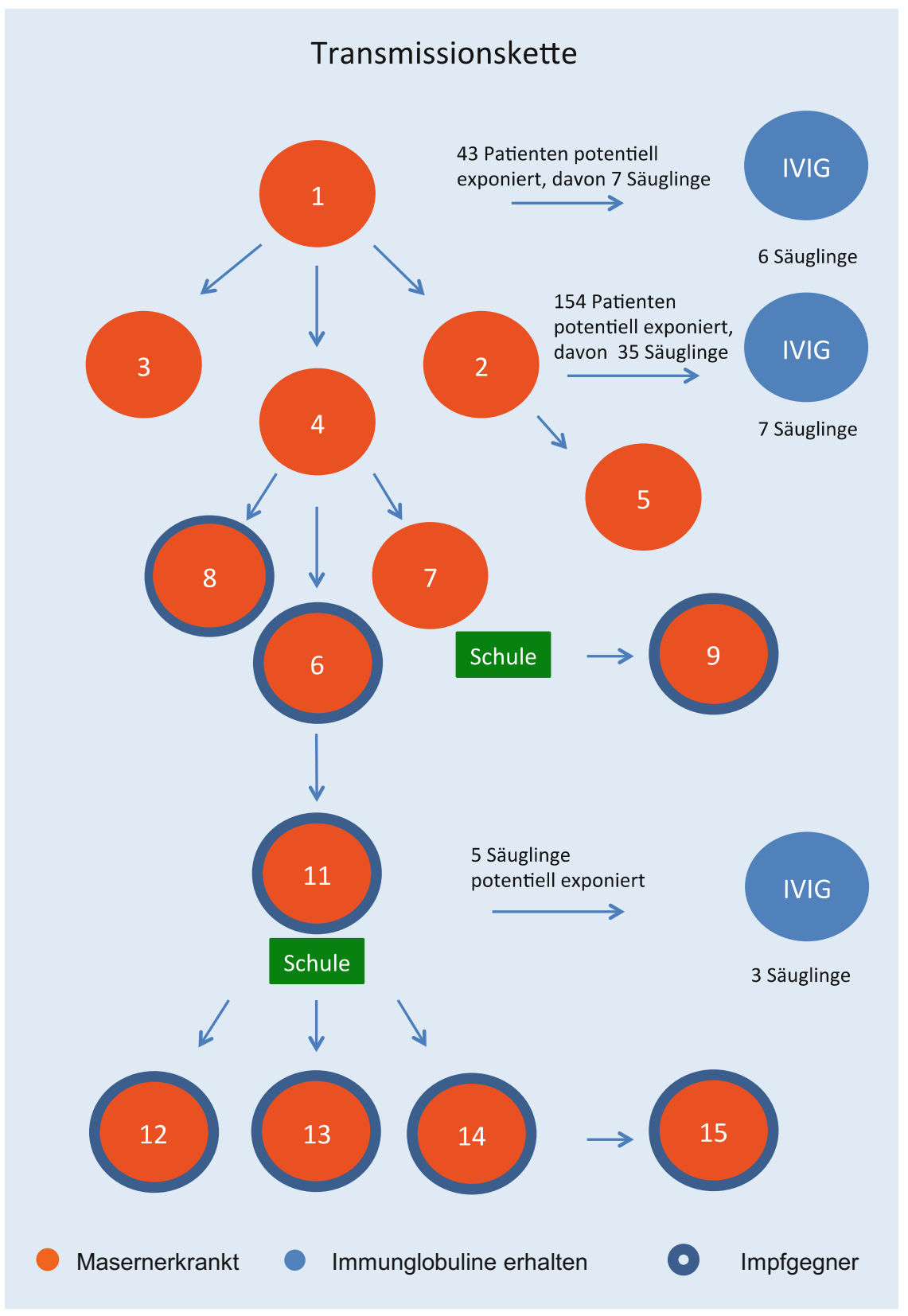

Abb. 1 A Transmissionsweg im Masernausbruch 2015. Weitere Fälle wurden aufgrund fehlender Quellenangabe nicht in der Abbildung berücksichtigt

die öffentlich bezahlt wurden, dokumentiert, somit auch die Masern-Mumps-Röteln(MMR)-Impfungen [13]. Statistisch wurde zur Signifikanztestung der Fisher's-Exakt-Test herangezogen.

\section{Beschreibung des Masern- ausbruches}

Im Zeitraum Februar 2015 bis Mai 2015 wurden in der Steiermark 19 Masernfälle bei Kindern und Jugendlichen beobachtet. Weiters wurden 8 erwachsene Pati- klinischen Untersuchung diagnostiziert wurden.

Aufgrund des Aufenthalts im allgemeinen Wartebereich wurden durch ihn 43 Patienten potenziell exponiert, davon sieben Säuglinge. Sechs dieser Säuglinge wurden stationär aufgenommen und erhielten intravenöse Immunglobuline (IVIG). Beim siebenten Säugling war die Exposition schon länger als sieben Tage her.

Insgesamt steckte der Indexpatient höchstwahrscheinlich drei weitere $\mathrm{Pa}$ tienten an: eine ungeimpfte Krankenschwesterschülerin $\langle 2\rangle$, einen Zivildiener $<3>$ und ein zweijähriges Kind $<4>$. Die Kinderkrankenschwesterschülerin arbeitete bis einen Tag vor Ausbruch des Masernexanthems (Masern serologisch gesichert) in der Ambulanz der Klinik. Bei der Annahme, dass sie vier Tage vor Beginn des Masernexanthems infektiös gewesen war, waren dadurch potenziell 154 Patienten (davon 35 Säuglinge) dem Masernvirus exponiert worden. Insgesamt erkrankte nach Rücksprache mit allen Exponierten glücklicherweise nur einer dieser 154 Personen klinisch an Masern. Dies war ein achtmonatiger Säugling, zu dem die Kinderkrankenschwesterschülerin am Vortag ihres Erkrankungsbeginnes Kontakt hatte $<5>$.

Der Zivildiener $<3>$ hatte nur eine MMR-Impfung erhalten und zeigte einen verspäteten Beginn sowie milden Verlauf der Masernerkrankung.

Das zweijährige Kind $<4>$ wurde einige Tage später erneut vorstellig, jedoch vergaß die Mutter den Masernverdacht trotz stattgefundener Aufklärung durch das Gesundheitsamt mitzuteilen. Das Kind war schwarzafrikanischer Abstammung und ein bei dieser Vorstellung durchgeführter Streptokokkenschnelltest fiel positiv aus. Das Masernexanthem wurde deswegen irrtümlicherweise als Scharlach interpretiert.

Dieses Kind steckte höchstwahrscheinlich drei weitere Kinder an. Ein sechsjähriges Kind $<6>$ nach Kontakt im Warteraum, das Geschwister $<7>$ von Patient 4 und ein Nachbarskind $<8>$. Das Geschwister $<7>$ steckte infolge höchstwahrscheinlich ein siebenjähriges Kind $<9>$ an, welches dieselbe Schulklasse besuchte. Das sechsjährige Kind, das 
in unserer Ambulanz infiziert worden war (6), steckte höchstwahrscheinlich ein weiteres Kind $<11>$ an, welches in derselben Straße wohnte.

Dieses wurde wegen Fiebers im Prodromalstadium der Masern beim niedergelassenen Kinderfacharzt vorstellig und wartete eine Stunde in dessen Warteraum. Dort hatte es Kontakt mit drei Säuglingen, die in weiterer Folge stationär aufgenommen wurden und prophylaktisch IVIG erhielten.

Patient 11 steckte höchstwahrscheinlich drei Klassenkameraden an $<12>$, $<13>,<14>$. Bei $<13>$ trat eine Otitis media als Komplikation der Masernerkrankung auf. Patient 14 wiederum steckte höchstwahrscheinlich sein Geschwister <15> an. - Abb. 1 zeigt den Zusammenhang der Transmissionskette.

In weiterer Folge traten Masernerkrankungen auf, bei welchen die eindeutige Zuteilung eines ursächlichen Ansteckenden fehlte:

- Ein zweijähriges Kind $<10>$ erkrankte, nachdem es sich bei einem Kinderfest, an dem auch Kinder mit floriden Masern teilgenommen hatten, angesteckt hatte.

- Unabhängig davon wurden bei einem Kindergartenfest, an dem ein an Masern erkranktes Kind teilnahm, ein fünfähriges Kind $<16>$ und sein sechsjähriges Geschwister $<17>$ infiziert.

- Eine Woche später wurde ein ungeimpfter 16-Jähriger <18> vorstellig, nachdem die Schulärztin ihn mit Masernverdacht an die Ambulanz überwies. Der Verdacht wurde serologisch bestätigt, jedoch konnte kein Ansteckungsort eruiert werden. Dieser steckte dann einen Schulkollegen aus der Nachbarklasse $<19>$ an, dessen Nichte nach Exposition dann stationär zur IVIG-Gabe aufgenommen werden musste, da sie noch ein Säugling war.

Elf Masernfälle wurden durch die Virologie Graz oder Virologie Wien serologisch oder mittels PCR gesichert, in acht Fällen wurden die Masern klinisch diagnostiziert.

Monatsschr Kinderheilkd 2019 · 167:46-50 https://doi.org/10.1007/s00112-017-0340-y

(c) Der/die Autor(en) 2017. Dieser Artikel ist eine Open-Access-Publikation.

\section{A. Trobisch · D. S. Klobassa · H. Gschiel · M. Wassermann-Neuhold · W. Zenz \\ Analyse des Masernausbruches 2015 in der Steiermark. Masernverbreitung erfolgte durch Impfgegner}

\section{Zusammenfassung}

2015 wurde in der Steiermark ein Masernausbruch bei Kindern und Jugendlichen mit 19 Fällen dokumentiert und retrospektiv deren Impfstatus und die Einstellung der Erziehungsberechtigten zum Impfen erhoben. Die Population der Masernerkrankten wurde mit der Population der steirischen Impfdatenbank verglichen und zeigte signifikant die präventive Wirkung der Masernimpfung. Zudem konnte unter den Nichtgeimpften der Masernpopulation eine Impfgegnerrate von $82 \%$ nachgewiesen werden. Es konnte eine dokumentierbare Transmissionskette mit 5 Kettengliedern gezeigt werden. Zudem konnten spezifische Probleme, die eine Übertragung begünstigen, beobachtet werden. Es werden Maßnahmen diskutiert, um Impflücken zu schließen und um zukünftige Ausbrüche zu verhindern.

Schlüsselwörter

Masern · Steiermark $\cdot 2015 \cdot$ Impfgegner . Impflücken

\section{Analysis of the $\mathbf{2 0 1 5}$ measles epidemic in Styria, Austria. Measles transmitted by anti-vaccinationists}

\section{Abstract}

In 2015, a measles outbreak amongst children occurred in Styria that totaled 19 cases, all of which were thoroughly documented. Retrospectively, the vaccination status and the guardian's vaccination opinion were investigated. The measles population was compared with the population of the Styrian vaccination database and significantly showed that the measles vaccination prevents the disease. Furthermore, an anti-vaccinationist rate of $82 \%$ was found in the unvaccinated measles population. A documentable chain of transmission with five chain-links was shown, as well as specific problems that facilitated outbreaks. Measures are discussed to close vaccination gaps and prevent future outbreaks.

\section{Keywords}

Measles · Styria $\cdot 2015 \cdot$ Anti-vaccination movement $\cdot$ Vaccination gaps

\section{Impfstatus und Einstellung der Eltern zur Masernimpfung}

17 von 19 Kindern waren vollkommen ungeimpft, zwei hatten nur eine Impfung, kein Kind hatte zwei Impfungen erhalten.

14/17 (82 \%) der ungeimpften Kinder waren aufgrund der impfkritischen Einstellung ihrer Eltern nicht geimpft worden. (Zwei Patienten waren ungeimpft, da sie erst kürzlich adoptiert worden waren, und ein Patient war über den eigenen Impfstatus falsch informiert worden.) Der Terminus „Impfgegner“ wurde hierbei als die grundsätzliche Ablehnung, (ohne medizinische oder religiöse Gründe) sein Kind impfen zu lassen, definiert.

Insgesamt musste 15 exponierten Säuglingen prophylaktisch IVIG verabreicht werden.
An Komplikationen wurde in diesem Masernausbruch lediglich eine Otitis media dokumentiert.

Die dokumentierte Impfrate in der Steiermark betrug seit Einführung der Impfdokumentation im Jahre 1999 schlechtestenfalls $86,69 \%$ (prozentuelle Rate für erste Teilimpfung für alle Kinder in der Steiermark des Jahrgangs 2002) [13]. Würde man nun annehmen, dass Impfgegner für die fehlenden Impfungen aller ungeimpften Kinder und Jugendlichen verantwortlich wären (was sicher nicht zutrifft), würde dies im schlimmsten Fall einer Impfgegnerrate von 13,31 \% entsprechen. Vergleicht man die Raten der Geimpften mit derjenigen der Ungeimpften der Masernerkrankten versus der Population der steirischen Impfdatenbank, zeigt sich mit statistischer Signifikanz $(p=0,0003)$, dass 


\begin{tabular}{|c|c|c|}
\hline & Nichtgeimpft & Geimpft \\
\hline Untersuchte Masernfälle 2015 & $17(89 \%)$ & 2 \\
\hline Steiermark gesamt Kohorte 2002 & $1547(13 \%)$ & 10.074 \\
\hline \multicolumn{3}{|c|}{ Fisher's-Exakt-Test: $p$-Wert $=0,00036,95 \% \mathrm{Cl}=13,11-493,59, \mathrm{OR}=55,35$} \\
\hline
\end{tabular}

die Masernimpfung verlässlich vor einer Erkrankung durch Masern schützt. In unserem Vergleich haben nichtgeimpfte Personen eine 55-mal höhere Wahrscheinlichkeit, an Masern zu erkranken, als geimpfte (• Tab. 1).

\section{Diskussion}

Wir beschreiben einen Masernausbruch mit 19 Fällen im Kindes- und Jugendalter in der Steiermark und zeigen, dass unzureichender Impfschutz zum Ausbruch und im Spitalsbereich zur Verbreitung führte und Impfgegner ein wesentlicher Faktor für die weitere Verbreitung der Masern in der Steiermark sind.

Ähnliche Ergebnisse zeigen sich auch in der Literatur. Bei zwei beschriebenen Masernepidemien 2005 in Deutschland waren 95-98\% ungeimpft [11].

Eine Literatursuche in den USA fand zwischen 2000 und 201518 publizierte Ausbrüche mit 1416 Masernfällen. Von 970 Masernfällen waren detaillierte Impfdaten verfügbar: 574 waren ungeimpft, 405 (70,6\%) gaben nichtmedizinische Gründe für die fehlende Impfung an [12].

Dass Impfgegner eine essenzielle Rolle in der Verbreitung der Masern spielen, wird anhand der hohen Rate von $82 \%$ unter den nichtgeimpften Masernerkrankten deutlich, vor allem auch im Vergleich zur tatsächlichen Impfgegnerrate in der Normalbevölkerung von etwa $4 \%$ laut Prof. Zwiauers Untersuchung [14]. Diese befragte in Niederösterreich die Einstellung von Eltern zum Thema Impfen. An dieser Erhebung waren 751 Eltern beteiligt (15\% Väter, $85 \%$ Mütter), wobei $4 \%$ (30 Elternteile) davon prinzipiell gegen Impfungen sind.

Besonders unverständlich ist die Tatsache, dass sich mehrere der beschriebenen Impfgegner bewusst gegen Schutzimpfungen entschieden, gleichzeitig jedoch im Prodromalstadium den Schul- mediziner aufgesucht haben und in allgemeinen Wartebereichen (zum Teil neben noch nicht impfbaren Säuglingen) auf dessen Behandlung gewartet haben.

Unser Bericht zeigt aber auch einige spezifische Probleme auf, welche die Weiterverbreitung der Masern begünstigen: Eine Masernerkrankung kann im Prodromalstadium nicht erkannt werden.

Da die Kinder in diesem Stadium schon klinisch krank sind, werden sie häufig beim Arzt vorgestellt.

Masern sind bei schwarzafrikanischen Kindern schwer zu diagnostizieren. Die serologische Maserndiagnostik liefert oft erst nach einigen Tagen einen eindeutigen Befund.

Ein weiteres spezifisches Problem betraf die Krankenschwesterschülerin, welche irrtümlicherweise bei der Einstellungsuntersuchung lediglich nach ihrem Immunstatus befragt wurde, anstatt wie empfohlen entweder die Serologie oder zwei dokumentierte Impfungen zu erheben [1].

Drei der exponierten Säuglinge konnten nicht mehr prophylaktisch mit IVIG behandelt werden, da bei der Krankenschwesterschülerin das Masernexanthem am Wochenende auftrat und die serologische Maserndiagnostik dadurch erst am Tag fünf nach Exanthembeginn vorlag. Somit waren fünf bis neun Tage seit der Exposition vergangen und die Sechstagefrist für eine prophylaktische IVIG-Gabe für die meisten Patienten überschritten $[1,5,7]$. Glücklicherweise hatte dieser Ausbruch nur eine Otitis media als Komplikation zu verzeichnen.

Wie sehr dieser Ausbruch die Bevölkerung verunsicherte, zeigte sich in der Tatsache, dass eine der beschriebenen Schulen für einen Tag geschlossen wurde, da die Lehrer bezüglich ihres eigenen Impfstatus in Bezug auf Masern unsicher waren und bekannt war, dass 15 Kinder an dieser Schule nicht gegen Masern geimpft waren und vier schon erkrankt waren.

Zusammenfassend beschreibt unsere Arbeit den Verlauf des Masernausbruchs bei 19 Kindern und Jugendlichen in der Steiermark und die essenzielle Rolle, welche Impfgegnern bei der Verbreitung der Erkrankung zukommt.

\section{Fazit für die Praxis}

Der Masernausbruch 2015 zeigt deutlich, dass in der Steiermark Impfgegner für die Verbreitung der Masern verantwortlich sind. Opfer sind die Jüngsten unserer Gesellschaft: 10 Säuglinge, allesamt aus Familien, die sich nicht gegen Impfungen aussprachen, mussten stationär aufgenommen werden, um nach einer Masernexposition in Warteräumen intravenöse Immunglobuline zu erhalten.

Eine Impfpflicht für Mitarbeiter im Gesundheitswesen wäre ein erster Schritt. Diese Maßnahme ist aber zu wenig, da die Verbreitung der Masern hauptsächlich durch Kinder von Impfgegnern erfolgte.

\section{Korrespondenzadresse

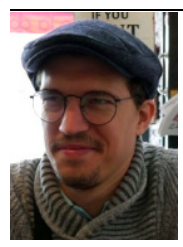 \\ Ass. Dr. A. Trobisch \\ Ambulanz für Vakzinologie und Infektionskrankheiten, Klinische Abteilung für Allgemeine Pädiatrie, Medizinische Universität Auenbruggerplatz 34/2, $8036 \mathrm{Graz}$, Österreich Andreas.Trobisch@ medunigraz.at}

Danksagung. Ein besonderer Dank gilt dem Land Steiermark, Abteilung 8 für Gesundheit, Pflege und Wissenschaft, Referat für Wissenschaft und Forschung als Fördergeber.

Funding. Open access funding provided by Medical University of Graz.

\section{Einhaltung ethischer Richtlinien}

Interessenkonflikt. A. Trobisch, D.S. Klobassa, H. Gschiel, M. Wassermann-Neuhold und W. Zenz geben an, dass kein Interessenkonflikt besteht.

Dieser Beitrag beinhaltet keine von den Autoren durchgeführten Studien an Menschen oder Tieren. 
Open Access. Dieser Artikel wird unter der Creative Commons Namensnennung 4.0 International Lizenz (http://creativecommons.org/licenses/by/4.0/deed. de) veröffentlicht, welche die Nutzung, Vervielfältigung, Bearbeitung, Verbreitung und Wiedergabe in jeglichem Medium und Format erlaubt, sofern Sie den/die ursprünglichen Autor(en) und die Quelle ordnungsgemäßnennen, einen Linkzur Creative Commons Lizenz beifügen und angeben, ob Änderungen vorgenommen wurden.

\section{Literatur}

1. American Academy of Pediatrics. Measles. In: Pickering LK, Baker CJ, Kimberlin DW, Long SS, eds. Red Book: 2015 Report of the Committee on Infectious Diseases. Elk Grove Village, IL: American Academy of Pediatrics; 2015:[535-547]

2. Center for Disease Control and Prevention (2015) Measles and Rubella Monitoring. April 2015.http:// ecdc.europa.eu/en/publications/Publications/ Measles-rubella-monitoring-second-quarter2015.pdf.Zugegriffen: 21.01.2017

3. Centers for Disease Control and Prevention (1999) Global measles control and regional elimination, 1998-1999. MMWR. Morbidity and mortality weekly report 48(49):1124-1130. https://www. cdc.gov/mmwr/preview/mmwrhtml/mm4849a3. htm. Zugegriffen:02.07.2017

4. World Health Organization Regional Office for Europe (2015) 4th Meeting of the European Regional Verification Commission for Measles and Rubella Elimination (RVC), The measles and rubella elimination target - the way forward after 2015, 26-29.10.2015 (S. 9-10). Copenhagen, Denmark. http://www.euro.who.int/_data/assets/pdf file/0011/304958/4th-RVC-meeting-report.pdf. Zugegriffen:02.07.2017

5. Barinaga JL, Skolnik PR (2013) Prevention and treatment of measles. JAMA:. doi:10.1001/jama. 1937.02780480049015

6. Kasper S, Holzmann H, Aberle SW, Gschiel H, Feenstra O, Allerberger F (2009) Measles outbreak in Styria, Austria, March-May 2009. Public Health 14(40):10-12

7. McLean HQ, Fiebelkorn AP, Temte JL, Wallace GS, Centers for Disease Control and Prevention (2013) Prevention of measles, rubella, congenital rubella syndrome, and mumps, 2013: summary recommendations of the Advisory Committee on Immunization Practices (ACIP). MMWR Recomm Rep62(RR-04):1-34

8. Muscat M, Mamou BM, Shefer A, Jankovic D, Deshevoy S, Butler R (2015) The state of measles and rubella in the WHO European region. Rev Esp Salud Publica 89(4):345-351. doi:10.4321/S113557272015000400002

9. Schmid D, Holzmann H, Schwarz K et al (2010) Measles outbreak linked to a minority group in Austria, 2008. Epidemiol Infect 138(3):415-425. doi:10.1017/S0950268809990604

10. Schmid D, Holzmann H, Abele S et al (2008) An ongoing multi-state outbreak of measles linked to non-immune anthroposophic communities in Austria, Germany, and Norway, March-April 2008. EuroSurveill 13(16):1-2

11. Siedler A, Tischer A, Mankertz A, Santibanez $S$ (2006) Two outbreaks of measles in Germany 2005. Euro Surveill 11(4):131-134

12. Phadke VK, Bednarczyk RA, Salmon DA, Omer SB (2016) Association between vaccine refusal and vaccine-preventable diseases in the United
States: a review of measles and pertussis. JAMA 315(11):1149-1158. doi:10.1001/jama.2016.1353

13. Wissenschaftliche Akademie für Vorsorgemedizin (2016) Abfrage Aus Der Steirischen Impfdatenbank (Stand Jänner 2016). Graz

14. Zwiauer K (2012/2013) Persönliche Mitteilung: Elternbefragung der Karl Landsteiner Gesellschaft für Pädiatrische Forschung und Fortbildung/Peri Human Relations GmbH zum Thema „Erheben von Wissen, Einstellungen und Verhaltensweisen von Eltern mit Kindern von 0 bis 13 Jahren rund um das Thema Impfen und Ernährung im Kindesalter" 12/2012-01/2013

\section{Genetik von Intelligenz und kognitiven Störungen}

Ein komplexes, aber relevantes Thema - nicht nur für die Humangenetik

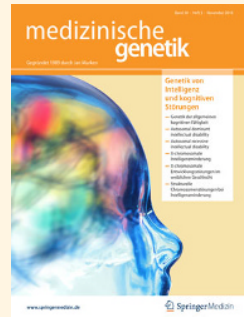

Intelligenz ist eines der bestuntersuchten Konstrukte der empirischen Verhaltenswissenschaften. Während der letzten Jahre haben die neuen Hochdurchsatz-Sequenzierungsverfahren zur Identifizierung von zahlreichen neuen Genen für die autosomal-dominante, autosomal-rezessive und X-gebundene Intelligenzminderung geführt.

Lesen Sie in Heft 3/2018 (November) der Zeitschrift medizinische genetik über die aktuellen Kenntnisse der Wissenschaft zum Thema Genetik von Intelligenz und kognitiven Störungen.

- Genetik der allgemeinen kognitiven Fähigkeit

- Autosomal dominante geistige Behinderung

- Autosomal rezessive geistige Behinderung

- X-chromosomale geistige Behinderung bei Jungen

- X-chromosomale geistige Behinderung bei Mädchen

- Strukturelle

Chromosomenaberrationen bei Intelligenzminderung

OPEN ACCESS - Diese Beiträge sind für Sie online frei verfügbar!

Suchen Sie noch mehr zum Thema? Mit e.Med - den maßgeschneiderten Fortbildungsabos von Springer Medizin - haben Sie Zugriff auf alle Inhalte von SpringerMedizin.de. Sie können schnell und komfortabel in den für Sie relevanten Zeitschriften recherchieren und auf alle Inhalte im Volltext zugreifen.

Weitere Infos zu e.Med finden Sie auf springermedizin.de unter "Abos" 